\title{
Small non-coding RNA STnc640 regulates expression of fimA fimbrial gene and virulence of Salmonella enterica serovar Enteritidis
}

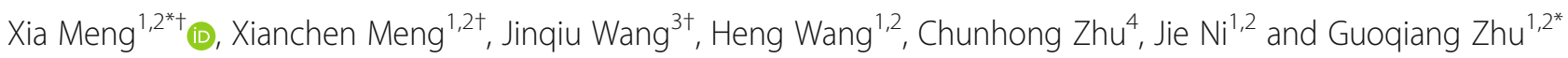

\begin{abstract}
Background: Small non-coding RNAs (sRNAs) regulate bacterial gene expression at the post-transcriptional level. STnc640 is a type of sRNA that was identified in Salmonella Typhimurium.

Results: In this study, STnc640 in Salmonella Enteritidis was confirmed to be an Hfq-dependent sRNA. TargetRNA software analysis showed that fimbrial genes fimA and bcfA were likely to be the target genes of STnc640. To investigate the target mRNAs and function of STnc640 in pathogenicity, we constructed the deletion mutant strain 50336 stnc640 and the complemented strain 50336stnc640/pstnc640 in Salmonella Enteritidis 50336. The RT-qPCR results showed that the mRNA level of fimA was decreased, while bcfA was unchanged in 50336 $\triangle$ stnc 640 compared with that in the wild type (WT) strain. The adhesion ability of 50336 $\Delta$ stnc640 to Caco-2 cells was increased compared to the 50336 WT strain. The virulence of $50336 \Delta$ stnc640 was enhanced in a one-day-old chicken model of $S$. Enteritidis disease as determined by quantifying the $50 \%$ lethal dose $\left(\mathrm{LD}_{50}\right)$ of the bacterial strains.
\end{abstract}

Conclusions: The results demonstrate that STnc640 contributes to the virulence of Salmonella Enteritidis.

Keywords: Salmonella Enteritidis, STnc640, Regulation, Adhesion, Virulence

\section{Background}

Small non-coding RNAs (sRNAs) in bacteria are stable transcripts approximately 50-500 nucleotides in length, often encoded in intergenic regions (IGRs), that play important roles in regulating gene expression at the post-transcriptional level [1-4]. sRNAs regulate many physiological processes, including metabolism, iron homeostasis, outer membrane protein biosynthesis, quorum sensing, and virulence [5-8]. Many of these sRNAs require the RNA-chaperone Hfq [9]. Nearly 100 distinct sRNAs have been identified in Salmonella [10].

Salmonella enterica serovar Enteritidis is an important Gram-negative intracellular pathogen with a broad host range. It can infect young chickens and cause symptoms

\footnotetext{
* Correspondence: mengxia_1@126.com; yzgqzhu@yzu.edu.cn

${ }^{+}$Xia Meng, Xianchen Meng and Jinqiu Wang contributed equally to this work.

${ }^{1}$ College of Veterinary Medicine, Yangzhou University, Yangzhou 225009,

China

Full list of author information is available at the end of the article
}

such as enteritis or systemic infection [11]. Adult chickens infected with Salmonella Enteritidis may have subclinical infections and become chronic carriers, leading to contamination of chicken meat and egg products and the resulting food-borne diarrheal illnesses in humans [12]. Adhesion to intestinal epithelial cells mediated by bacterial fimbriae is a necessary first step for colonization [13-16]. Whole-genome sequencing has identified 13 fimbriae operons in the Salmonella Enteritidis strain P125109 [17]. The fim operon directs the assembly of type I fimbriae, which are involved in reproductive tract infection and in egg contamination [15]. Type I fimbriae and other multiple fimbrial adhesins are also required for the colonization of the intestinal lumen and for the virulence of Salmonella Typhimurium in mice [18].

STnc640 is a novel Hfq-binding sRNA that was identified in Salmonella Typhimurium through deep sequencing and transcriptomic analysis of Hfq-bound sRNAs and mRNAs [19]. Here we constructed a stnc640 deletion

(c) The Author(s). 2019 Open Access This article is distributed under the terms of the Creative Commons Attribution 4.0 International License (http://creativecommons.org/licenses/by/4.0/), which permits unrestricted use, distribution, and reproduction in any medium, provided you give appropriate credit to the original author(s) and the source, provide a link to the Creative Commons license, and indicate if changes were made. The Creative Commons Public Domain Dedication waiver (http://creativecommons.org/publicdomain/zero/1.0/) applies to the data made available in this article, unless otherwise stated. 
mutant and characterized the role of this sRNA in bacterial adhesion and virulence.

\section{Results}

Hfq plays a positive role on STnc640 stability

To determine whether the stability of STnc640 depends on the sRNA chaperone protein Hfq, the abundance of stnc640 transcripts in S. Enteritidis WT strain 50336, mutant $50336^{\triangle} h f q$ and the complemented mutant $50336 \triangle h f q / \mathrm{p} h f q$ were determined using RT-qPCR. The abundance of stnc640 was significantly reduced in $50336 \triangle h f q$, exhibiting only about $2 \%$ of that in the WT strain $(P<0.01)$ and was restored in the $50336 \triangle h f q / \mathrm{p} h f q$ mutant (Fig. 1). This indicated that Hfq played a positive role on STnc640 stability.

\section{Candidate mRNA targets of STnc640}

Candidate mRNA targets of STnc640 were predicted using TargetRNA2 [20]. There were nine consecutive hybridization seeds between the AU-rich region of STnc640 (nts 263-277) and bcfA (nts 37-51). There were 11 consecutive hybridization seeds between the coding sequences (codons 8-25) of fimA mRNA and STnc640 (codons 99-125).

\section{Construction and growth characteristics of the mutant} $50336 \triangle$ stnc640 and complemented strain $50336 \triangle$ stnc640/ pstnc640

S. Enteritidis strain 50336 contains an stnc640 gene with $97 \%$ identity to the $S$. Typhimurium strain LT2 stnc640 gene. STnc640 was located in a non-coding region between

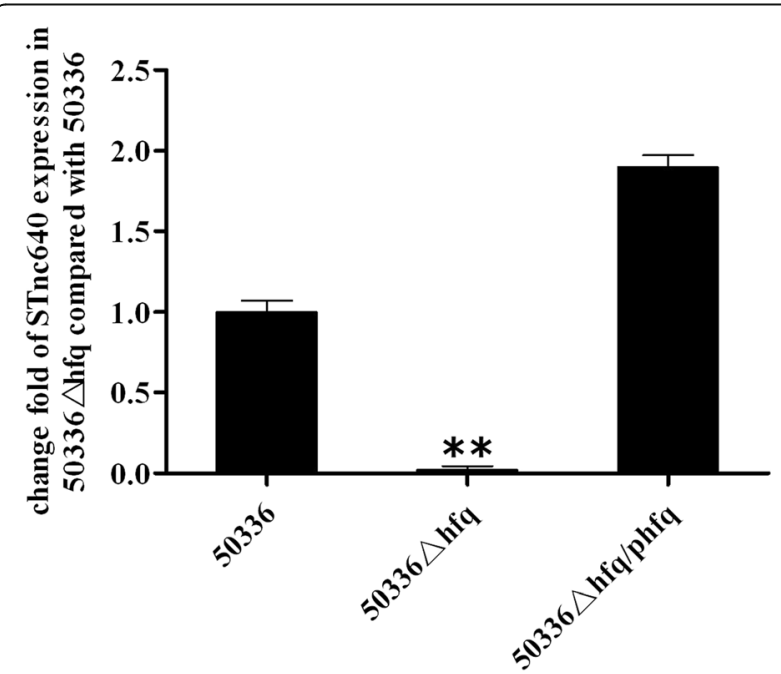

Fig. 1 Fold changes of the STnc640 gene mRNA level were measured in the mutant 50336 $\mathrm{hfq}$ and complementation strain $50336 \Delta h f q / p h f q$ by RT-qPCR compared with the wild-type $S$. Enteritidis 50336. Assays were performed in triplicate. ${ }^{* *}$ Indicates statistically significant difference compared with the wild type strain $(p<0.01)$ the genes SEN1810 and icdA in S. Enteritidis. In the construction of the deletion and the complemented strains, a 460 bp DNA fragment of the non-coding region was deleted and complemented. We constructed an stnc640 deletion mutant $50336{ }^{\triangle}$ stnc 640 and compared its growth to the WT and complemented strains. The growth rate of $50336^{\triangle} \operatorname{sinc} 640$ was significantly reduced during the $\log$ phase from $2 \mathrm{~h}$ to $3 \mathrm{~h}(P<0.05)$ (Fig. 2$)$.

\section{STnc640 regulates fimA expression and affects adherence} and invasion to Caco- 2 cells

To determine whether $b c f A$ and/or fimA expression are regulated by STnc640, we quantified $b c f A$ and fimA expression using RT-qPCR. The fimA but not $b c f A$ transcript abundance was reduced in the $\Delta \operatorname{stnc} 640$ mutant compared with the WT strain (Fig. 3). To investigate whether deleting stnc640 affected bacterial adhesion and invasion by regulating fim $A$, we performed bacterial adhesion and invasion assays. $\Delta s t n c 640$ was enhanced in adhering and invading to Caco-2 cells compared with the WT strain (Fig. 4).

\section{Deleting stnc640 enhances virulence in chickens}

$\mathrm{LD}_{50}$ assays were performed to analyze the effect of stnc640 on S. Enteritidis virulence in chickens. All of the chickens displayed intestinal hyperemia and diarrhea 10 $h$ post infection. Higher mortality appeared when infected by $50336^{\triangle} \operatorname{sinc} 640$ compared to the WT strain and the complemented strain. The mortality rates for $10^{7}, 10^{8}$ and $10^{9} \mathrm{CFU}$ bacteria treatment were 5,57 and $95 \%$ separately when infected by $50336^{\triangle}$ stnc 640 . The mortality rates for the above three dose treatment were 5, 50 and $85 \%$ separately when infected by the WT

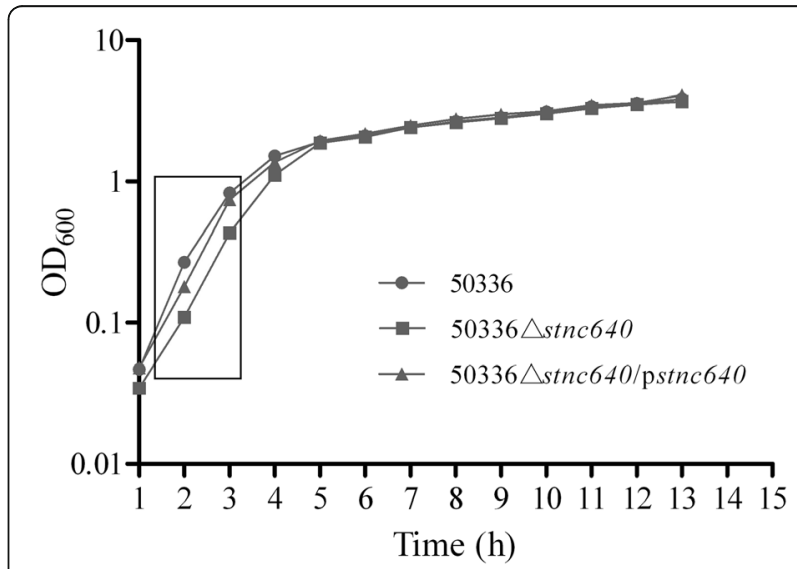

Fig. 2 Growth curves of wild-type S. Enteritidis 50336, mutant $50336 \triangle \operatorname{stnc} 640$, and complementation strain 50336 $\Delta$ stnc640/ pstnc640. $\mathrm{OD}_{600}$ values of triplicate cultures in $L B$ medium were determined at $1 \mathrm{~h}$ intervals. Data are the means of three independent experiments. The box in the figure indicates that the growth was significantly reduced from $2 \mathrm{~h}$ to $3 \mathrm{~h}$ 


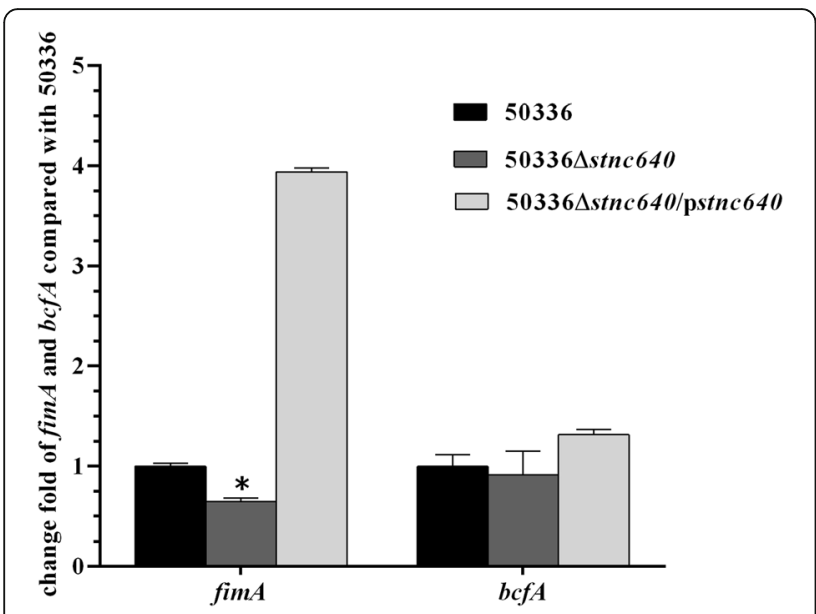

Fig. 3 The mRNA levels of fimbrial genes fimA and bcfA were determined in the mutant 50336 $\triangle$ stnc640 and 50336 $\triangle$ stnc640/ pstnc640 compared to wild-type S. Enteritidis 50336 by qRT-PCR. Assays were performed in triplicate

strain, and the rates were 0,5 and $62 \%$ when infected by the complemented strain. The LD50s were calculated 14 days post-infection. The LD50 values of the WT strain 50336, $50336^{\triangle}$ stnc 640 and $50336^{\triangle}$ stnc640/pstnc640 were $2.9 \times 10^{8}, 2.0 \times 10^{8}$ and $5.1 \times 10^{8} \mathrm{CFU}$, respectively. This indicated that the virulence of $50336^{\triangle}$ stnc 640 was enhanced approximately 1.5 -fold compared with the WT. The virulence of complemented strain $50336{ }^{\triangle}$ stnc640/pstnc640 has attenuated compared with the WT strain and the $50336^{\triangle} \operatorname{stnc} 640$ mutant. Tests of isolation and identification of bacteria showed that all

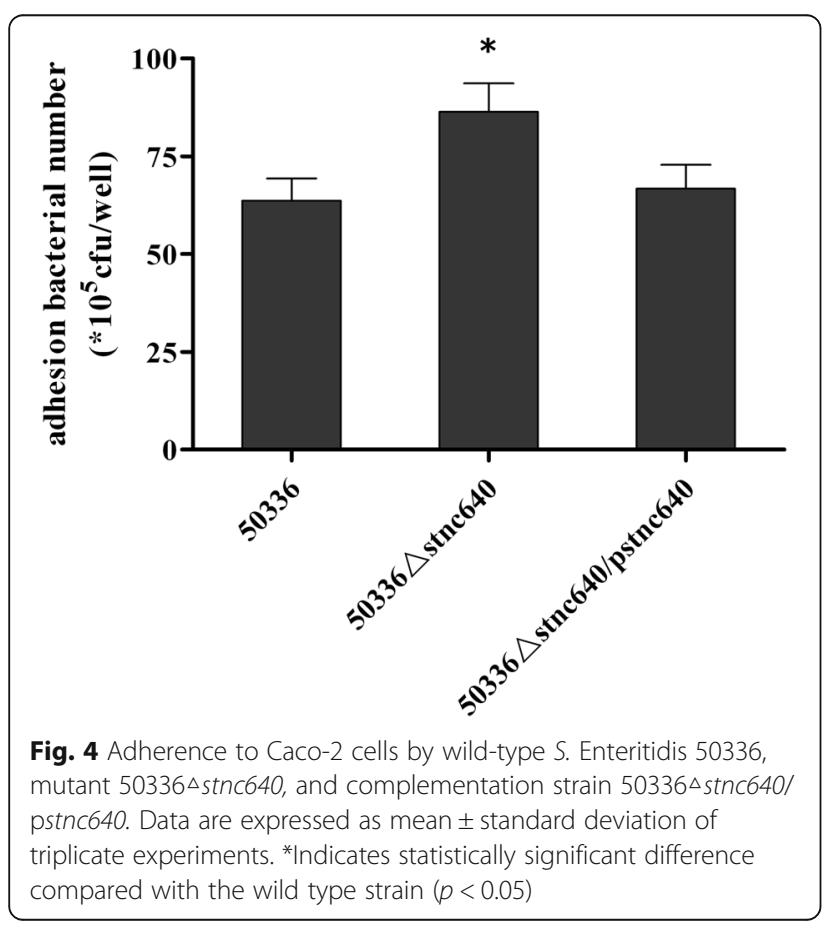

three strains of S. Enteritidis were widely distributed in the liver, spleen, and caecum of the infected chickens.

\section{Discussion}

sRNAs are a ubiquitous class of molecules that can regulate gene expression at the post-transcriptional level. Most sRNAs can interact with their target mRNAs by basepairing actions and then modulate translation, degradation, or stability of mRNA [4]. In this study, an sRNA gene $\operatorname{stnc} 640$ of $S$. Enteritidis strain 50336 was cloned and showed $97 \%$ identity with stnc640 of S. Typhimurium. This indicated that stnc640 has very high homology within the genus. Identification of the STnc640 target gene is important for the study of sRNA function. To date, the target genes and the function of STnc640 remain unknown. We thus identified likely candidate mRNA targets of STnc640 (fim $A$ and $b c f A$ ) by bioinformatics predication technology using TargetRNA2.

The growth rates of the WT strain, $50336^{\triangle}$ stnc640, and $50336 \triangle$ stnc $640 /$ pstnc640 were determined by measuring $\mathrm{OD}_{600}$. The growth rate of $50336^{\triangle} \operatorname{stnc} 640$ was lower than those of the WT strain and $50336^{\triangle}$ stnc640/pstnc640 in the log phase. Many sRNAs can directly sense multiple environmental signals such as fluctuations in temperature, $\mathrm{pH}$, and metabolites [3, 21]. The deletion of STnc640 apparently weakened environmental adaptation, leading to the decline in growth rate at the log phase, but the final concentration of bacteria was not affected.

The STnc640 candidate targets $\operatorname{fim} A$ and $b c f A$ were verified by detecting their mRNA levels by RT-qPCR. The expression of fimA was down-regulated in $50336^{\triangle}$ stnc 640 compared to the WT strain. This suggested that that STnc640 could regulate fimA expression. In other words, fimA was a likely target of STnc640. However, the regulation mechanism needs further study. FimA is a major fimbrial subunit in Salmonella enterica. The Type I fimbriae can alter virulence of S. Typhimurium toward mice [18]. Type I fimbriae are also involved in clearance of $S$. Enteritidis from the blood and in egg contamination by $S$. Enteritidis in laying hens [15]. Deletion of STnc640 led to a decrease of fimA expression, but the ability of adhesion to Caco-2 cells of the STnc640 mutant was stronger than that of the wild type strain. This indicated that there is no direct relationship between fim $A$ expression and adhesion ability. Rajashekara found that deletion of the fim $A$ gene in $S$. Enteritidis did not affect the ability to invade Caco-2 cells and colonize the chicken caecum [22], which is consistent with our result. Multiple fimbrial adhesins are required for Salmonella colonization of the chicken intestine tract. We supposed that up-regulation of other adhesion-related genes expression, but not down-regulation of the fimA gene, caused the adhesion ability enhancement in the STnc640 deletion mutant. Adhesion to and colonization of host cells are important factors for 
virulence. In our study, the STnc640 deletion in S. Enteritidis strengthened the ability to adhere to Caco-2 cells and thus increased the virulence in chickens. We inferred that STnc640 could inhibit $S$. Enteritidis virulence by affecting adhesion. For further confirm of whether STnc640 could inhibit virulence, overexpression of STnc640 in the wild type strain and comparison that with wild type need to be performed in the future.

\section{Conclusions}

Small non-coding RNA STnc640 could regulate the expression of fimA fimbrial gene in $S$. Enteritidis. The deletion of STnc640 in $S$. Enteritidis strengthened the ability to adhere to and colonize in Caco-2 cells and thus increased the virulence in chickens. It was supposed that STnc640 could inhibit $S$. Enteritidis virulence by affecting adhesion.

\section{Methods}

\section{Bacterial strains, plasmids and cell culture conditions}

The bacteria strains and plasmids used in this study are listed in Table 1. Salmonella Enteritidis wild type (WT) strain 50336, the mutants $50336 \triangle{ }^{\triangle} \operatorname{stnc} 640$ and $50336 \triangle h f q$, complemented mutants $50336^{\triangle} \operatorname{sinc640/pstnc640}$ and $50336 \triangle h f q / \mathrm{p} h f q$, and E.coli DH5 $\alpha$ were grown in Luria-Bertani broth (LB) or on LB agar plates at $37^{\circ} \mathrm{C}$. Strains containing temperature-sensitive plasmids such as pCP20 or pKD46 were grown at $30^{\circ} \mathrm{C}$. Strains harboring antibiotic resistance were cultured in LB containing $100 \mu \mathrm{g} / \mathrm{ml}$ of Ampicillin (Amp) or $34 \mu \mathrm{g} / \mathrm{ml}$ of chloramphenicol (Cm) when appropriate. To determine growth rates, the strains were grown at $37^{\circ} \mathrm{C}$ with agitation $(180 \mathrm{rpm})$ in LB broth, and the optical density at $600 \mathrm{~nm}\left(\mathrm{OD}_{600}\right)$ was measured every hour. Human colorectal adenocarcinoma epithelial cells (Caco-2) were cultured as described previously [23].

\section{Stability detection of STnc640 in hfq mutants}

$S$. Enteritidis WT strain 50336, the mutant $50336 \triangle h f q$, and the complemented mutant $50336^{\triangle} h f q / \mathrm{p} h f q$ were grown to an $\mathrm{OD}_{600}$ of 2.5 and collected by centrifugation. Total RNA was extracted and reverse transcribed to cDNA. The mRNA transcripts of stnc640 in WT 50336, 50336 ${ }^{\circ} h f q$, and $50336 \triangle h f q / \mathrm{p} h f q$ were detected by real-time quantitative PCR (RT-qPCR) using primers stnc640-F and stnc640$\mathrm{R}$.

\section{Prediction of candidate mRNA targets of STnc640}

Candidate mRNA targets of STnc640 were predicted using TargetRNA2 [20] (http://old-tempest.wellesley. edu/ btjaden/TargetRNA2/index.html.oldtempest). Using this website, we selected the Salmonella Enteritidis strain P125109 genome, input the STnc640 sequence, and then specified 90 nucleotides upstream and 30 nucleotides downstream of the translation start sites of candidate targets. Candidate targets were identified by specifying at least nine consecutive hybridization seeds corresponding to an initial interaction between the sRNA and mRNA with a $p$-value below 0.01 .

\section{Construction of the stnc640 deletion mutant and the complemented strain}

The primers used are listed in Table 2. The stnc640 gene was cloned using PCR primers that flank the stnc640 gene in Salmonella Typhimurium. The construction of stnc640negative mutants of $S$. Enteritidis 50336 was generated by the phage $\lambda$-Red-mediated recombination system as described previously $[24,25]$. Primers P3 and P4 were used to amplify chloramphenicol resistance-encoding genes to construct the first recombinant strain $50336 \triangle$ stnc640::cat. The stnc640 complete deletion mutant $50336 \triangle$ stnc 640 was confirmed by PCR using primers (P1, P2) and sequencing

Table 1 Bacterial strains and plasmids used in this study

\begin{tabular}{|c|c|c|}
\hline Strains/plasmids & Characteristics & References \\
\hline \multicolumn{3}{|l|}{ Strains } \\
\hline CMCC(B)50336 & Salmonella enterica serovar Enteritidis wild-type & NICPBP, China \\
\hline $50336 \triangle \operatorname{stnc} 640$ & stnc640 deficient mutant & This study \\
\hline $50336 \triangle \operatorname{stnc} 640 / p \operatorname{stnc} 640$ & $50336 \Delta$ stnc640 carrying pBR-stnc640 (Ampr) & This study \\
\hline $50336 \Delta h f q$ & hfq deficient mutant & [23] \\
\hline $50336 \Delta h f q / p h f q$ & $50336 \Delta h f q$ carrying pBR-hfq $\left(\mathrm{Amp}^{r}\right)$ & [23] \\
\hline \multicolumn{3}{|l|}{ Plasmids } \\
\hline pKD3 & $\mathrm{Cm}^{r} ; \mathrm{Cm}$ cassette teplate & [24] \\
\hline pKD46 & Amp ${ }^{r}, \lambda$ Red recombinase expression & [24] \\
\hline pCP20 & $\mathrm{Amp}^{r}, \mathrm{Cm}^{r}$; Flp recombinase expression & {$[24]$} \\
\hline pBR-stnc640 & pBR322 carrying the full stnc640 gene (Amp') & This study \\
\hline pGEM-T Easy & cloning vector, Ampr & Takara \\
\hline pMD19 T-simple & cloning vector, Ampr ${ }^{r}$ & Takara \\
\hline
\end{tabular}


Table 2 Primers for the PCR and the size of PCR products

\begin{tabular}{lll}
\hline Primer & Sequence $\left(5^{\prime}-3^{\prime}\right)$ & Size $($ bp $)$ \\
\hline P1 & TGGAAATGGCGGACATCT & $809 / 416$ \\
P2 & TAAAGTCAACCCAGGCTCC \\
P3 & GAAATGTAGTGAGTTGGTGACGCGATTATCGCAAATATGTAATAACGATGTGTAGGCTGGAGCTGCTTCG \\
P4 & AACCTTATACTTCCACTATGGCAGATAGGTTGAGCATATGTCTCCTGACATATGAATATCCTCCTTAG \\
P5 & CCCAAGCTTCGATTATCGCAAATATGCGA \\
P6 & GCGTCGACTCAGCAGTCTCTATTAAAGCA \\
bcfA-F & TGACGCTGCCTGTTCTGTT \\
bcfA-R & GCAGTCTTCCAGTTTGATGGTG \\
fimA-F & GACTGCGATCCGAAAGTGG \\
fimA-R & CAGAGGAGACAGCCAGCAAA \\
gyrA-F & GCATGACTTCGTCAGAACCA \\
gyrA-R & GGTCTATCAGTTGCCGGAAG & 1114 \\
\hline
\end{tabular}

the PCR product that contained the primers $\mathrm{P} 3$ and $\mathrm{P} 4$ sequences and lacked of stnc640 sequences using fluorescence-based chain-termination method with a DNA sequencer ABI 3730XL. The complemented strain was generated by cloning the full-length stnc 640 gene into plasmid pBR322, which was transferred to the stnc640 mutant. The mutant $50336^{\triangle} h f q$ and complemented mutant $50336 \triangle h f q / \mathrm{p} h f q$ were described previously [23].

\section{RNA extraction and real-time quantitative PCR}

Bacteria were grown to an $\mathrm{OD}_{600}$ of 2.5 in $\mathrm{LB}$ medium and collected by centrifugation. Total RNA was extracted using TRIzol reagent (Invitrogen, NY, USA). cDNA was synthesized using the PrimeScript RRT reagent kit with gDNA Eraser (Takara Bio, Shiga, Japan). Transcript abundance was quantified using RT-qPCR with SYBR Premix Ex Taq II (Takara) and the primers listed in Table 2 using an ABI7500 instrument (Applied Biosystems, USA). Assays were performed in triplicate, and all data were normalized to the endogenous reference gene gyrA using the. $2^{-\triangle \triangle C T}$ method [26].

\section{Bacterial adherence and invasion assays}

Bacterial adherence and invasion assays were performed as described previously [27]. Bacteria were incubated with a monolayer of $1 \times 10^{5}$ Caco- 2 cells at a multiplicity of infection (MOI) of 100 at $37^{\circ} \mathrm{C}$ in 96-well tissue culture plates (Corning) for $2 \mathrm{~h}$. Infections were carried out in triplicate. Infected cell monolayers were gently washed three times with PBS to remove loosely adherent bacteria. Cells were lysed with $0.5 \%$ Triton X-100 for $30 \mathrm{~min}$. The lysates were serially diluted and plated onto LB agar plates for the enumeration of adherent and invaded bacteria.

\section{Animal infections}

One-day-old chickens (National Chickens Genetic Resources, Yangzhou, China) were randomly divided into one control group and three infection groups $(n=20,10 \mathrm{fe}-$ males and 10 males). Salmonella Enteritidis strains 50336,

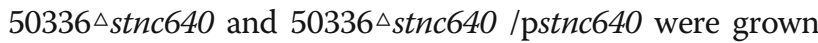
to early stationary phase with an $\mathrm{OD}_{600}$ of 2.5 in LB medium, harvested by centrifugation, washed, and resuspended to $5 \times 10^{7} \mathrm{CFU} / \mathrm{mL}, 5 \times 10^{8} \mathrm{CFU} / \mathrm{mL}$ and $5 \times 10^{9}$ $\mathrm{CFU} / \mathrm{mL}$ gradient suspensions in sterile PBS prior to inoculation into infection group chickens. Three infection groups were separately inoculated with $200 \mu \mathrm{L} 5 \times 10^{7}$ $\mathrm{CFU} / \mathrm{mL}, 5 \times 10^{8} \mathrm{CFU} / \mathrm{mL}$ or $5 \times 10^{9} \mathrm{CFU} / \mathrm{mL}$ bacterial suspensions, while the control group received $200 \mu \mathrm{L}$ PBS by subcutaneous injection. Signs of chickens illness and death were monitored daily. The $50 \%$ lethal dose $\left(\mathrm{LD}_{50}\right)$ was calculated 14 days post-infection using the method described previously [23]. Briefly, the numbers of dead and surviving chickens were recorded daily. The summation of cumulative dead and surviving chickens of each dose was taken. The $\mathrm{LD}_{50}$ was calculated using the data on percent mortality using the arithmetical method of Reed and Muench [28]. All live chickens were euthanized by pentobarbital after the assays. All procedures complied with institutional animal care guidelines and were approved by the Animal Care and Ethics Committee of the Yangzhou University (Approval ID: YZUDWSY2017-0026).

\section{Statistical analysis}

Data were analyzed using Student's $t$ test for independent samples. Differences were considered significant if $P \leq 0.05$.

\footnotetext{
Abbreviations

Amp: Ampicillin; Caco-2: Human colorectal adenocarcinoma epithelial cells; Cm: Chloramphenicol; IGRs: Intergenic regions; LB: Luria-Bertani broth; $\mathrm{LD}_{50}$ : 50\% lethal dose; $\mathrm{OD}_{600}$ : Optical density at $600 \mathrm{~nm}$; RT-qPCR: Real-time quantitative PCR; sRNAs: Small non-coding RNAs; WT: Wild type
}

\section{Acknowledgments}

We thank LetPub (www.letpub.com) for its linguistic assistance during the preparation of this manuscript. 


\section{Authors' contributions}

XiaM, GZ and JW conceived, designed and drafted the manuscript. XiaM and XianM performed the majority of the experiments. HW helped with experiments and provided valuable discussion and modified the final manuscript. CZ and JN participated in experimental procedures and data analysis. All authors read and approved the final manuscript.

\section{Funding}

This study was supported by grants from the National Key R \& D Program (2017YFD0500203), Chinese National Science Foundation (Nos. 31101826 and 31672579), A Project Funded by the Priority Academic Program Development of Jiangsu Higher Education Institutions (PAPD), Jiangsu High Education Science Foundation (No.14KJB230002), State Key Laboratory of Veterinary Biotechnology (No.SKLVBF201509) and Nature Science Foundation Grant of Yangzhou (No.YZ2014019). The funding bodies did not play direct roles in the design of the study and collection, analysis, and interpretation of data and in writing the manuscript.

\section{Availability of data and materials}

The datasets used and analysed during the current study are available from the corresponding author on reasonable request.

\section{Ethics approval and consent to participate}

One-day-old chickens were provided by the National Chickens Genetic Resources in Yangzhou of China. The protocol was approved by the Animal Care and Ethics Committee of Yangzhou University (Approval ID: YZUDWSY2017-0026).

\section{Consent for publication}

Not applicable.

\section{Competing interests}

The authors declare that they have no competing interests.

\section{Author details \\ ${ }^{1}$ College of Veterinary Medicine, Yangzhou University, Yangzhou 225009, China. ${ }^{2}$ Jiangsu Co-innovation Center for Prevention and Control of Important Animal Infectious Diseases and Zoonoses, Yangzhou 225009, China. ${ }^{3}$ Department of Animal Husbandry and Veterinary Medicine, Beijing Vocational College of Agriculture, Beijing 102442, China. ${ }^{4}$ Jiangsu provincial key lab for genetics and breeding of poultry, Jiangsu Institute of Poultry Science, Yangzhou 225125, China.}

Received: 10 February 2019 Accepted: 27 August 2019

Published online: 05 September 2019

\section{References}

1. Hebrard M, Kroger C, Srikumar S, Colgan A, Handler K, Hinton JC. sRNAs and the virulence of Salmonella enterica serovar typhimurium. RNA Biol. 2012;9:437-45.

2. Majdalani N, Vanderpool CK, Gottesman S. Bacterial small RNA regulators. Crit Rev Biochem Mol Biol. 2005;40:93-113.

3. Vogel J. A rough guide to the non-coding RNA world of Salmonella. Mol Microbiol. 2009:71:1-11.

4. Waters LS, Storz G. Regulatory RNAs in bacteria. Cell. 2009;136:615-28.

5. Bejerano-Sagie $M$, Xavier KB. The role of small RNAs in quorum sensing. Curr Opin Microbiol. 2007;10:189-98.

6. Gripenland J, Netterling S, Loh E, Tiensuu T, Toledo-Arana A, Johansson J. RNAs: regulators of bacterial virulence. Nat Rev Microbiol. 2010;8:857-66.

7. Masse E, Salvail H, Desnoyers G, Arguin M. Small RNAs controlling iron metabolism. Curr Opin Microbiol. 2007;10:140-5.

8. Papenfort K, Vogel J. Regulatory RNA in bacterial pathogens. Cell Host Microbe. 2010;8:116-27.

9. Chao Y, Vogel J. The role of Hfq in bacterial pathogens. Curr Opin Microbiol. 2010;13:24-33.

10. Sridhar J, Papenfort K, Vogel J. The small RNAs of Salmonella. Salmonella from genome to function. New York: Horizon press; 2011. p. 123-48.

11. Saeed AM, Gast RK, Potter ME, Wall PG. Salmonella enterica serovar Enteritidis in humans and animals: epidemiology, pathogenesis, and control. Ames: lowa State University Press; 1999.
12. De Buck J, Van Immerseel F, Haesebrouck F, Ducatelle R. Colonization of the chicken reproductive tract and egg contamination by Salmonella. J Appl Microbiol. 2004;97:233-45.

13. Baumler AJ, Tsolis RM, Heffron F. The Ipf fimbrial operon mediates adhesion of Salmonella typhimurium to murine Peyer's patches. Proc Natl Acad Sci U S A. 1996:93:279-83.

14. Baumler AJ, Tsolis RM, Heffron F. Contribution of fimbrial operons to attachment to and invasion of epithelial cell lines by Salmonella typhimurium. Infect Immun. 1996;64:1862-5.

15. De Buck J, Van Immerseel F, Haesebrouck F, Ducatelle R. Effect of type 1 fimbriae of Salmonella enterica serotype Enteritidis on bacteraemia and reproductive tract infection in laying hens. Avian Pathol. 2004;33:314-20.

16. Weening EH, Barker JD, Laarakker MC, Humphries AD, Tsolis RM, Baumler AJ. The Salmonella enterica serotype typhimurium Ipf, bcf, stb, stc, std, and sth fimbrial operons are required for intestinal persistence in mice. Infect Immun. 2005;73:3358-66.

17. Thomson NR, Clayton DJ, Windhorst D, Vernikos G, Davidson S, Churcher C, et al. Comparative genome analysis of Salmonella Enteritidis PT4 and Salmonella Gallinarum 287/91 provides insights into evolutionary and host adaptation pathways. Genome Res. 2008;18:1624-37.

18. Van der Velden AW, Baumler AJ, Tsolis RM, Heffron F. Multiple fimbrial adhesins are required for full virulence of Salmonella typhimurium in mice. Infect Immun. 1998;66:2803-8.

19. Sittka A, Lucchini S, Papenfort K, Sharma CM, Rolle K, Binnewies TT, et al. Deep sequencing analysis of small noncoding RNA and mRNA targets of the global post-transcriptional regulator, Hfa. PLoS Genet. 2008:4:e1000163.

20. Kery MB, Feldman M, Livny J, Tjaden B. (2014) TargetRNA2: identifying targets of small regulatory RNAs in bacteria. Nucleic Acids Res. 2014:42:124-9.

21. Klinkert B, Narberhaus F. Microbial thermosensors. Cell Mol Life Sci. 2009;66:2661-76.

22. Rajashekara G, Munir S, Alexeyev MF, Halvorson DA, Wells CL, Nagaraja KV. Pathogenic role of SEF14, SEF17, and SEF21 fimbriae in Salmonella enterica serovar enteritidis infection of chickens. Appl Environ Microbiol. 2000;66:1759-63.

23. Meng X, Meng X, Zhu C, Wang H, Wang J, Nie J, et al. The RNA chaperone $\mathrm{Hfq}$ regulates expression of fimbrial-related genes and virulence of Salmonella enterica serovar Enteritidis. FEMS Microbiol Lett. 2013:346:90-6.

24. Datsenko KA, Wanner BL. One-step inactivation of chromosomal genes in Escherichia coli K-12 using PCR products. Proc Natl Acad Sci U S A. 2000;97:6640-5

25. Duan Q, Zhou M, Zhu X, Yang Y, Zhu J, Bao W, et al. Flagella from F18+ Escherichia coli play a role in adhesion to pig epithelial cell lines. Microb Pathog. 2013;55:32-8.

26. Livak KJ, Schmittgen TD. Analysis of relative gene expression data using real-time quantitative PCR and the 2(-Delta Delta C(T)) method. Methods. 2001;25:402-8.

27. Jouve M, Garcia MI, Courcoux P, Labigne A, Gounon P, Le Bouguenec C. Adhesion to and invasion of HeLa cells by pathogenic Escherichia coli carrying the afa-3 gene cluster are mediated by the AfaE and AfaD proteins, respectively. Infect Immun. 1997:65:4082-9.

28. Reed $\sqcup$, Muench $H$. Asimple method of estimating fifty percent end points. Am J Hyg. 1938;27:493-7.

\section{Publisher's Note}

Springer Nature remains neutral with regard to jurisdictional claims in published maps and institutional affiliations.

Ready to submit your research? Choose BMC and benefit from:

- fast, convenient online submission

- thorough peer review by experienced researchers in your field

- rapid publication on acceptance

- support for research data, including large and complex data types

- gold Open Access which fosters wider collaboration and increased citations

- maximum visibility for your research: over $100 \mathrm{M}$ website views per year

At $\mathrm{BMC}$, research is always in progress.

Learn more biomedcentral.com/submissions 